\title{
Factores que controlan las propiedades texturales de arcillas intercaladas con disoluciones de circonio y aluminio
}

\author{
A. GIL ${ }^{1}$, M.A. VICENTE ${ }^{2}$ y L.M. GANDIA ${ }^{1}$ \\ ${ }^{1}$ Departamento de Química Aplicada, Edificio Los Acebos, Universidad Pública de Navarra, 31006 Pamplona, España \\ ${ }^{2}$ Departamento de Química Inorgánica, Facultad de Ciencias Químicas, Universidad de Salamanca, 37008 Salamanca, España
}

En este trabajo se ha estudiado la evolución de las propiedades texturales de una saponita y una montmorillonita intercaladas con disoluciones de circonio y aluminio tras calcinación a $500{ }^{\circ} \mathrm{C}$. Los sólidos presentaron un diferente comportamiento en función de la naturaleza de la disolución de intercalación y de la arcilla empleada. Tras calcinación, la superficie específica y el volumen microporoso de las arcillas intercaladas con disoluciones de aluminio disminuyeron de manera importante, lo cual se ha relacionado con las distancias interlaminar e interpilar que presentaron los materiales intercalados. Por contra, las superficies específicas de las arcillas intercaladas con disoluciones de circonio aumentaron, probablemente gracias al efecto combinado de la descomposición térmica de los ligandos orgánicos unidos al circonio en los policationes y la posible presencia de partículas mesoporosas de $\mathrm{ZrO}_{2}$ sobre la superficie de los sólidos.

Palabras clave: arcillas intercaladas, distancia interlaminar, distancia interpilar, adsorción de nitrógeno.

Factors controlling the textural properties of zirconia and alumina pillared clays

The texture development of a saponite and a montmorillonite, intercalated with zirconium and aluminium oligomers, after calcination at $500{ }^{\circ} \mathrm{C}$ has been studied. Remarkable differences were observed among samples, depending on the source of the intercalating polycations and the parent material. On calcining, the alumina pillared clays suffered noticeable specific surface area and micropore volume losses, which have been related to the interlayer and interpillar distances of the samples. On the contrary, the zirconia pillared clays showed a slight surface area increase, which affected both micropores and mesopores and could be due to the combined effect of the thermal decomposition of the zirconium oligomers and the possible presence of mesoporous $\mathrm{ZrO}_{2}$ particles.

Key words: pillared clays, interlayer distance, interpillar distance, nitrogen adsorption.

\section{INTRODUCCIÓN}

Las arcillas laminares intercaladas con polihidroxicationes metálicos pueden considerarse como una nueva generación de materiales con una estructura tridimensional microporosa de dimensiones moleculares. Tras calcinación, se genera una estructura estable que se caracteriza por las distancias entre las láminas de la arcilla (distancia interlaminar) y entre los óxidos intercalados (distancia interpilar) (Figura 1). La distancia interlaminar depende de la naturaleza química y del tamaño de las especies intercaladas. La distancia interpilar está relacionada



Figura 1.- Estructura bidimensional de una arcilla laminar intercalada con polihidroxicationes metálicos. Se definen algunos de sus parámetros característicos. con la cantidad de especies intercaladas y su distribución. Este reparto está afectado por la extensión y distribución de la densidad de carga en la lámina de las arcillas (1), así como por efectos estéricos ligados al tamaño de las especies intercaladas. Ambas distancias, interlaminar e interpilar, pueden modificarse durante los tratamientos térmicos implicados en la síntesis si se produce la eliminación de ligandos orgánicos, así como por la deshidratación y sinterizado de los polihidroxicationes metálicos (2-9).

La distancia interlaminar se calcula a partir de la medida experimental del espaciado basal, d(001), por difracción de rayos $\mathrm{X}$, al que se resta el espesor de la lámina del material. Los cationes poliméricos $\mathrm{u}$ oligoméricos obtenidos por hidrólisis básica de sales de $\mathrm{Al}, \mathrm{Zr}$, Ti, Cr, Fe y Ga son los más empleados en el proceso de intercalación. Estos sistemas presentan espaciados basales entre 16 y $28 \AA$, que corresponden a espaciados interlaminares entre 6 y $18 \AA$.

La distancia interpilar es más difícil de determinar y de controlar. Se ha propuesto que este parámetro es el que contribuye en mayor medida a la distribución de tamaños de microporos en las arcillas intercaladas (10). Esta distancia depende del tamaño y de la cantidad de especies intercaladas entre las láminas de la arcilla. A su vez, la cantidad de especies intercaladas depende directamente de la cantidad de cationes metáli- 
cos intercambiables que presenta la arcilla, representada por la capacidad de intercambio catiónico (CIC). En tal caso, la CIC puede llegar a ser un factor clave si se quiere optimizar la síntesis de las arcillas intercaladas con respecto a una adecuada distribución de los polihidroxicationes metálicos (11). En efecto, este aspecto ha sido convenientemente demostrado por Mori y col. (12-14), quienes modificaron la CIC de una montmorillonita por tratamiento térmico controlado tras intercambio catiónico de la arcilla con disoluciones de $\mathrm{Li}^{+}, \mathrm{Co}^{2+}$ y $\mathrm{Ni}^{2+}$. Sus resultados indicaron que se require un mínimo valor de CIC en el material laminar para obtener una arcilla intercalada térmicamente estable; esto implica la necesidad de un número mínimo de polihidroxicationes en el espaciado interlaminar. Por otro lado, un excesivo valor de CIC puede causar un efecto negativo debido a la presencia de un gran número de especies que reducen la accesibilidad al entramado poroso. A estas mismas conclusiones se puede llegar a partir de los trabajos de Jones y Purnell (15) y Sychev y col. (16), en donde se describe la intercalación de montmorillonitas con disoluciones de aluminio y de cromo, respectivamente. Con la misma intención, también se ha estudiado la intercalación de saponitas sintéticas de carga superficial variable (carga comprendida entre 0,8 y 1,5 por cada 22 átomos de oxígeno presentes en la celda unidad) con polihidroxicationes de aluminio. Lambert y col. (1722) han encontrado una distribución máxima igual a una especie intercalada por cada 5,9 celdas unidad que está controlada por efectos estéricos. En aquellas saponitas que presentaban una mayor carga superficial, y que a priori debería permitir la intercalación de un mayor número de polihidroxicationes, la cantidad de aluminio retenido permaneció constante. Incluso el origen de la carga, debida a sustituciones isomórficas en la capa tetraédrica, o a sustituciones isomórficas o vacantes catiónicas en la capa octaédrica, afecta a la facilidad con que se intercala una arcilla.

Con respecto a la distancia interpilar, Clearfield y col. (23) han realizado cálculos para una montmorillonita intercalada con disoluciones de aluminio bajo dos supuestos: $i$. los polihidroxicationes $\left(\mathrm{Al}_{13} \mathrm{O}_{4}(\mathrm{OH})_{24}\left(\mathrm{H}_{2} \mathrm{O}\right)_{12}\right)^{7+}\left(\mathrm{Al}_{13}\right)$ mantienen su carga característica de la disolución $(+7)$ cuando se introducen entre las láminas, y ii. se hidrolizan para generar un polihidroxicatión de carga +4 cuando se introducen entre las láminas de la arcilla. Considerando valores de CIC entre 150 y 50 meq/100 g de arcilla, los autores obtienen valores de distancias interpilar entre 5,6 y $14,8 \AA$, cuando se supone que el catión presenta una carga de +7 , y entre 9,8 y $21,8 \AA$, si la carga fuese +4 .

La distancia interpilar puede resultar también notablemente afectada por ciertas variables del proceso de síntesis, como son la cantidad de polihidroxicatión metálico añadido con respecto a la masa de arcilla presente (razón cantidad de policatión/ arcilla) (24-26) y la composición de la disolución de intercalación (27-30). El efecto de la razón cantidad de policatión/arcilla es muy similar al que se encuentra con la CIC de la arcilla. De hecho, un valor bajo de esta variable se traduce en una baja estabilidad térmica del sólido resultante, y una excesiva concentración de policatión reduce la accesibilidad a la estructura porosa (26). Desde el punto de vista de la disolución de intercalación, tanto el grado de polimerización como la carga del polihidroxicatión metálico están controlados por la concentración de la sal metálica en disolución, la razón de hidrólisis (o el pH), así como por la temperatura y el tiempo de envejecimiento. La influencia de estas variables ha sido ampliamente estudiada para la hidrólisis de $\mathrm{AlCl}_{3}(27,28,30)$, donde además del polihidroxicatión $\mathrm{Al}_{13}$ implicado normalmente en la inter- calación de arcillas, se ha sugerido la presencia de otras especies formadas por condensación y crecimiento lineal $(29,30)$ del catión $\mathrm{Al}_{13}$, haciendo difícil el cálculo de la carga efectiva de las especies intercaladas. El grado de polimerización de un catión metálico, y consiguientemente la carga efectiva de los policationes formados, no son fáciles de controlar. Algunos autores como Poncelet y col. (31) han resuelto este problema midiendo la CIC que presentan las arcillas tras el proceso de intercalación con disoluciones de aluminio. Las medidas indicaron que la carga neutralizada por los polihidroxicationes intercalados está comprendida entre el 43 y el $82 \%$ de la CIC original de la arcilla, lo que permite calcular la carga efectiva de cada policatión $\mathrm{Al}_{13}$, aunque con la suposición de que ésta sea la única especie intercalante. En la intercalación con disoluciones de circonio y titanio, hidrolizadas con disoluciones de $\mathrm{HCl}$, las especies que normalmente se consideran responsables del proceso de intercalación son los policationes $\left(\mathrm{Zr}_{4}(\mathrm{OH})_{8}\left(\mathrm{H}_{2} \mathrm{O}\right)_{16} \mathrm{Cl}_{\mathrm{z}}\right)^{(8-\mathrm{z})+}$ y $\left((\mathrm{TiO})_{8}(\mathrm{OH})_{12}\right)^{4+}$, respectivamente, aunque en estos cationes el proceso de polimerización es aún más complejo que en el $\mathrm{Al}$, y se han propuesto también otros policationes con estructuras bidimensionales y tridimensionales $(32,33)$, que presentan una amplia distribución de cargas y de tamaños.

La calcinación a una temperatura relativamente alta es la última etapa de la preparación de estos materiales, transformando los polihidroxicationes metálicos intercalados entre las láminas en pilares óxidos. La temperatura a la que se realiza esta calcinación tiene, por ello, una gran influencia en las propiedades texturales de los sólidos finales (34). Lógicamente, la elección de la temperatura de calcinación debe tener en cuenta la estabilidad térmica de los pilares, siendo $500{ }^{\circ} \mathrm{C}$ la temperatura más frecuentemente utilizada. La calcinación a una temperatura demasiado elevada provoca la degradación de la microestructura de la arcilla intercalada y, en consecuencia, una pérdida del volumen microporoso. Este comportamiento se ha relacionado con la deshidroxilación de las especies inicialmente intercaladas y con una progresiva degradación tanto del catión intercalado como de la arcilla (35-38). Ge y col. (1) también han descrito el proceso como el resultado de una redistribución y reestructuración de los polihidroxicationes intercalados, pudiéndose generar una estructura microporosa más uniforme.

En este trabajo se analizan y comparan las estructuras microporosas de una saponita y una montmorillonita intercaladas con disoluciones de circonio y aluminio, deducidas a partir de las isotermas de adsorción a bajas presiones relativas de $\mathrm{N}_{2}$ a 77 K. Se dedica una atención especial a los conceptos de distancia interlaminar y distancia interpilar.

\section{PROCEDIMIENTO EXPERIMENTAL}

En este trabajo se han utilizado las fracciones menores de $2 \mu \mathrm{m}$ de dos arcillas laminares naturales, una saponita (Ballarat, California, EEUU) y una montmorillonita (Gador, Almería, España). Ambas arcillas presentaron una capacidad de intercambio catiónico (CIC) de 0,90 y 1,17 meq/g, respectivamente. Como disoluciones de intercalación se emplearon una disolución de circonio en ácido acético (Sigma-Aldrich) y una disolución de $\mathrm{AlCl}_{3} \cdot 6 \mathrm{H}_{2} \mathrm{O}$ hidrolizada con $\mathrm{NaOH}(\mathrm{OH} / \mathrm{Al}=2,2$; $\mathrm{pH}=4,1 ; 24$ horas a temperatura ambiente). En síntesis diferentes, se añadieron 8 gramos de cada arcilla laminar natural sobre las disoluciones anteriormente preparadas, siendo las razones de intercalación $20 \mathrm{mmol} \mathrm{Zr} / \mathrm{g}$ arcilla y $5 \mathrm{mmol} \mathrm{Al} / \mathrm{g}$ 
arcilla, respectivamente. Tras un periodo de contacto, a temperatura ambiente, de 2 horas (para las disoluciones de $\mathrm{Zr}$ ) o de 24 horas (para las disoluciones de $\mathrm{Al}$ ), las suspensiones se lavaron por centrifugación y diálisis, secandose a $50{ }^{\circ} \mathrm{C}$ durante 16 horas. Las arcillas intercaladas se calcinaron finalmente a $500{ }^{\circ} \mathrm{C}$ durante 4 horas (34). La nomenclatura utilizada es BAsap-Zr y GAmont-Zr, para las arcillas intercaladas con las disoluciones de circonio, y BAsap-Al y GAmont-Al, cuando se emplearon disoluciones de aluminio. La temperatura de calcinación $\left({ }^{\circ} \mathrm{C}\right)$ también ha sido incluída en la denominación de las arcillas.

La adsorción de $\mathrm{N}_{2}$ se llevó a cabo a $77 \mathrm{~K}$ empleando un equipo volumétrico estático (Micromeritics ASAP 2010). Las arcillas se desgasificaron previamente a $200{ }^{\circ} \mathrm{C}$ durante 24 horas. Todos los análisis se realizaron empleando 0,2 $\mathrm{g}$ de muestra y añadiendo cantidades sucesivas de nitrógeno iguales a $4 \mathrm{~cm}^{3} \mathrm{STP} / \mathrm{g}$ hasta una presión parcial $\left(\mathrm{p} / \mathrm{p}^{\circ}\right)$ igual a 0,01 y a partir de este valor se dosifica automáticamente el suficiente volumen de $\mathrm{N}_{2}$ como para alcanzar una tabla preestablecida de 39 valores de presión relativa. El tiempo de equilibrio de cada uno de los puntos de la isoterma de adsorción en este intervalo fue de al menos dos horas.

Los espaciados basales, $\mathrm{d}(001)$, se determinaron mediante difracción de rayos X (Siemens D-500) empleando el método de polvo microcristalino.

\section{RESULTADOS Y DISCUSIÓN}

Las isotermas de adsorción de nitrógeno de las arcillas intercaladas se presentan en las Figuras 2 y 3. Las isotermas de adsorción son del tipo I según la clasificación propuesta por Brunauer, Deming, Deming y Teller (BDDT) (39), que son características de sólidos microporosos. Los parámetros que caracterizan las propiedades texturales de las arcillas y que se han calculado a partir de las isotermas de adsorción se han resumido en la Tabla I. La intercalación con los polihidroxicationes de circonio y aluminio produjo un importante aumento de la superficie específica y del volumen de poros total y microporoso, con respecto de los valores obtenidos para las arcillas de partida. La calcinación a $500{ }^{\circ} \mathrm{C}$ de las arcillas intercaladas dió lugar a interesantes cambios texturales que se pueden resumir en los siguientes puntos:

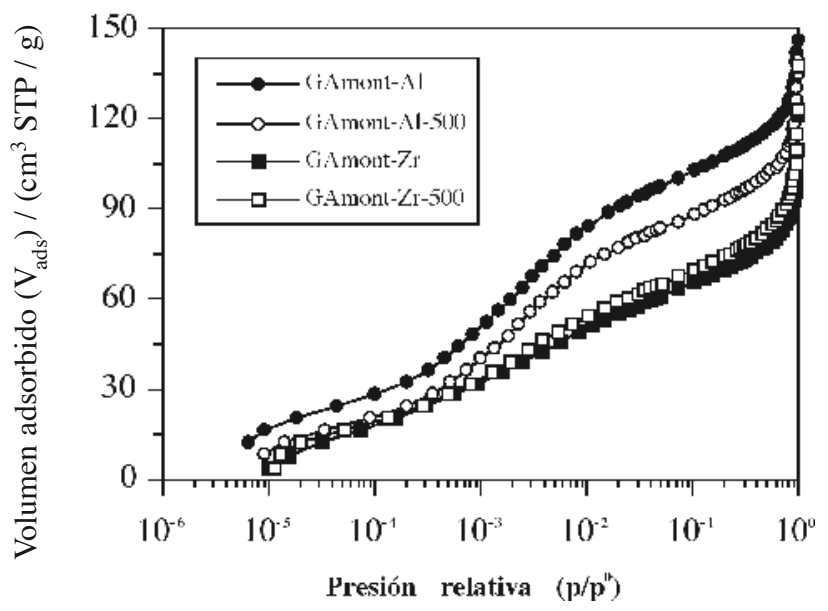

Figura 2.- Isotermas de adsorción de $\mathrm{N}_{2}$ desde bajas presiones relativas a $77 \mathrm{~K}$ de la montmorillonita Gador (GAmont) intercalada con disoluciones de circonio (GAmont-Zr) y aluminio (GAmont-Al). i.- El área superficial de las arcillas intercaladas con disoluciones de circonio aumentó en pequeña proporción, siendo más importante el aumento al emplear la saponita como arcilla de partida. Este efecto, como puede observarse en las isotermas presentadas en las Figuras 1 y 2, afecta a todo el rango de presiones relativas. Desde el punto de vista del volumen de poros, una importante contribución se debe a los mesoporos, como puede deducirse a partir de los volúmenes de poros total y microporoso presentados en la Tabla I.

ii.- Contrariamente a la evolución observada en las arcillas intercaladas con disoluciones de circonio, la superficie específica de las arcillas intercaladas con disoluciones de aluminio disminuyó, y de manera más significativa cuando se empleó la saponita como arcilla de partida. Como en el caso anterior, esta modificación afectó a todo el rango de presiones relativas (véase Figura 2 y Figura 3). Por otro lado, en esta serie de muestras, es el volumen microporoso el que se vió afectado en una mayor extensión (véase Tabla I).

En un trabajo previo (40), se han presentado las evidencias que indican que las disoluciones empleadas en la intercalación no alteran la estructura de las arcillas. En tal caso, las propiedades texturales desarrolladas por las arcillas intercaladas se deben exclusivamente a la presencia de polihidroxicationes metálicos en el espaciado interlaminar. A continuación se van a discutir los principales factores que pueden controlar la evolución durante la calcinación de los parámetros que caracterizan las propiedades texturales de las arcillas intercaladas.

Como se ha explicado en el apartado de introducción, la estructura microporosa de las arcillas intercaladas se caracteriza por la distancia entre las láminas de arcilla y la distancia entre las especies intercaladas.

La distancia interlaminar se calcula a partir de la medida experimental del espaciado basal, d(001), por difracción de rayos $X$, al que se resta el espesor de la lámina del material. Los espaciados basales medidos para las arcillas intercaladas objeto de este trabajo fueron respectivamente 20,3 $\AA$ (BAsap-


$\AA$ (GAmont-Zr-500). Estos resultados son similares a los que se han presentado en la bibliografía para la intercalación de arcillas con disoluciones de circonio y aluminio, lo que confirma el éxito del proceso. Además, teniendo en cuenta los espesores de las láminas de las arcillas de partida utilizadas, los resultados

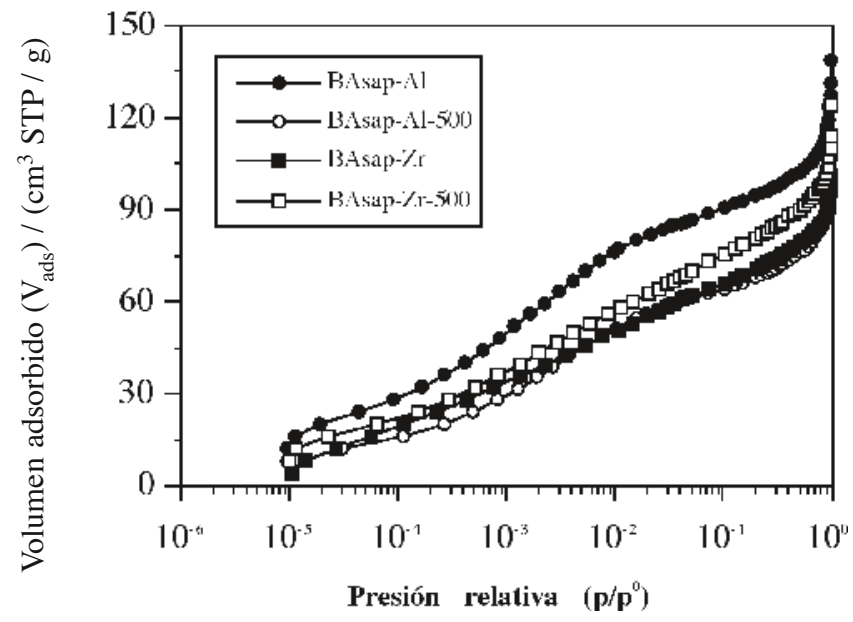

Figura 3.- Isotermas de adsorción de $\mathrm{N}_{2}$ desde bajas presiones relativas a $77 \mathrm{~K}$ de la saponita Ballarat (BAsap) intercalada con disoluciones de circonio (BAsap-Zr) y aluminio (BAsap-Al). 
también indican que las distancias interlaminares alcanzadas son similares cuando se emplean polihidroxicationes de circonio o de aluminio en la intercalación.

Las superficies específicas de las arcillas intercaladas con disoluciones de aluminio presentaron valores muy elevados, siendo $392 \mathrm{~m}^{2} / \mathrm{g}$ para la muestra BAsap-Al y $443 \mathrm{~m}^{2} / \mathrm{g}$ para la muestra GAmont-Al, respectivamente. Como se ha indicado anteriormente, durante la calcinación a $500{ }^{\circ} \mathrm{C}$ se produce una importante pérdida de superficie, siendo más importante para la saponita y que afecta a los microporos de ambas arcillas (véase Tabla I). Este comportamiento ha sido descrito por algunos autores (34-38) como el resultado de una deshidroxilación inicial de las especies intercaladas y una progresiva degradación de la arcilla y del policatión intercalado. Ge y col. (1) también han relacionado esta evolución con una redistribución lateral y reestructuración de las especies intercaladas, generándose al mismo tiempo una estructura microporosa más uniforme. Si se compara el comportamiento de los dos materiales de partida, BAsap-Al y GAmont-Al, la calcinación a $500{ }^{\circ} \mathrm{C}$ reduce parcialmente sus propiedades texturales, presentando la montmorillonita mayor estabilidad térmica que la saponita. Este resultado puede relacionarse con la distribución de los polihidroxicationes de aluminio entre las láminas de ambas arcillas. Dado que las disoluciones de aluminio empleadas en la intercalación de ambas arcillas tenían la misma composición, parece razonable pensar que como la montmorillonita muestra una mayor CIC, debe admitir una mayor cantidad de especies intercaladas, lo que se traduce en una mejor resistencia térmica tras el proceso de intercalación.

Contrariamente a la evolución descrita para las arcillas intercaladas con disoluciones de aluminio, la calcinación a $500{ }^{\circ} \mathrm{C}$ prácticamente no produjo modificaciones en la superficie específica y en el volumen de poros de las arcillas intercaladas con disoluciones de circonio. No obstante, si la comparación se realiza en función de la composición de las disoluciones de intercalación, la superficie específica de la montmorillonita intercalada con disoluciones de aluminio (GAmont-Al-500) resultó muy superior a la obtenida cuando se empleó la disolución de circonio (GAmont-Zr-500). En el caso de la saponita, no se observa una diferencia significativa entre las superficies específicas al emplear diferentes disoluciones de intercalación. En este sentido hay que indicar que a partir de las estructuras propuestas para los polihidroxicationes de circonio resultantes de la hidrólisis del acetato de circonio (41), el aumento observado en la superficie específica tras el tratamiento térmico puede deberse en parte a la descomposición térmica de los ligandos acetato, lo que puede facilitar el acceso a la estructura porosa de las arcillas intercaladas. Por otra parte, de acuerdo con los valores de volúmenes de poros total y microporoso presentados en la Tabla I, puede observarse una importante contribución del volumen de mesoporos. Este hecho, unido con la gran cantidad de circonio presente en las arcillas intercaladas $\left(36,4\right.$ y $27,4 \%$ en peso como $\mathrm{ZrO}_{2}$ para BAsap-Zr-500 y GAmont-Zr-500, respectivamente) sugiere que ambos resultados pueden estar relacionados. Sorprendentemente, la mayor cantidad de $\mathrm{ZrO}_{2}$ corresponde a la arcilla BAsap-Zr-500, que es la que presenta un menor valor de CIC. En tal caso, se debe considerar la cristalinidad de las arcillas como otro factor a tener en cuenta en el proceso de intercalación. Se ha descrito $(31,42,43)$ que la cristalinidad de las arcillas favorece el proceso de intercalación debido a una mejor separación de las láminas. Dado que la saponita de Ballarat es más cristalina que la montmorillonita de Gador (2), se puede justificar de esta forma la mayor cantidad de $\mathrm{Zr}$ presente en la saponita intercalada con
TABla I. Propiedades teXturales Calculadas A PARTIR DE LAS ISOTERMAS DE ADSORCIÓN DE $\mathrm{N}_{2}$ A $77 \mathrm{~K}$.

\begin{tabular}{|l|c|c|c|c|}
\hline & $\mathrm{d}(001)(\AA)$ & $\mathrm{A}_{\text {Lang }}{ }^{\mathrm{a}}\left(\mathrm{m}^{2} / \mathrm{g}\right)$ & $\mathrm{V}_{\mathrm{p}}{ }^{\mathrm{b}}\left(\mathrm{cm}^{3} / \mathrm{g}\right)$ & $\mathrm{V}_{\mu \mathrm{p}-\mathrm{t}}{ }^{\mathrm{c}}\left(\mathrm{cm}^{3} / \mathrm{g}\right)$ \\
\hline BAsap & 13,8 & $70\left(\mathrm{C}^{\mathrm{d}}=229\right)$ & 0,139 & 0,011 \\
BAsap-500 & 10,9 & $48(\mathrm{C}=240)$ & 0,130 & 0,004 \\
BAsap-Al & - & $392(\mathrm{C}=500)$ & 0,214 & 0,138 \\
BAsap-Al-500 & 20,3 & $280(\mathrm{C}=363)$ & 0,178 & 0,096 \\
BAsap-Zr & $12,6-22,7$ & $287(\mathrm{C}=275)$ & 0,167 & 0,111 \\
BAsap-Zr-500 & 20,5 & $324(\mathrm{C}=276)$ & 0,191 & 0,126 \\
Gamont & 16,1 & $138(\mathrm{C}=279)$ & 0,166 & 0,038 \\
GAmont-500 & 10,6 & $127(\mathrm{C}=282)$ & 0,176 & 0,030 \\
GAmont-Al & - & $443(\mathrm{C}=428)$ & 0,233 & 0,158 \\
GAmont-Al-500 & 19,9 & $380(\mathrm{C}=401)$ & 0,216 & 0,134 \\
GAmont-Zr & $13,9-17,0$ & $283(\mathrm{C}=291)$ & 0,187 & 0,101 \\
GAmont-Zr-500 & 18,3 & $299(\mathrm{C}=324)$ & 0,213 & 0,105 \\
\hline
\end{tabular}

a.- Superficie específica a partir del modelo de Langmuir $(0,01 \leq \mathrm{p} /$ $\left.\mathrm{p}^{0} \leq 0,05\right)$.

b.- Volumen de poros total a $\mathrm{p} / \mathrm{p}^{0}=0,99$.

c.- Volumen microporoso obtenido a partir del método $t$, empleando para el cálculo del espesor de la capa de moléculas de $\mathrm{N}_{2}$ adsorbidas la expresión de Harkins y Jura. Area de la molécula de $\mathrm{N}_{2}, 16,2 \AA^{2}$ [39].

d.- Constante $\mathrm{C}$, característica de la intensidad de las interacciones adsorbato-adsorbente.

respecto a la montmorillonita. No obstante, también es posible que durante la síntesis parte del circonio se deposite fuera del espacio interlaminar, dando lugar, tras calcinación, a partículas mesoporosas de óxido de circonio que contribuyan de manera significativa al incremento observado en el volumen de poros total de ambas arcillas intercaladas con este elemento, aunque la presencia de partículas de $\mathrm{ZrO}_{2}$ no ha podido ser confirmada por difracción de rayos $\mathrm{X}$.

\section{CONCLUSIONES}

En este trabajo se ha estudiado la evolución textural con la temperatura de calcinación de las propiedades de una saponita y una montmorillonita intercaladas con disoluciones de circonio y aluminio. Las superficies específicas y los volúmenes de microporos de las arcillas intercaladas con disoluciones de aluminio disminuyeron de manera importante tras la calcinación a $500{ }^{\circ} \mathrm{C}$, siendo este efecto más importante en el caso de la saponita. Este comportamiento se ha interpretado como consecuencia de una mayor estabilidad térmica de la montmorillonita intercalada con respecto de la saponita. Además de otros posibles factores, es probable que esta mejor resistencia térmica de la montmorillonita se deba a una mayor cantidad de especies intercaladas presentes como consecuencia de su mayor CIC. Contrariamente a la evolución que se observa en las arcillas intercaladas con disoluciones de aluminio, la superficie específica de las arcillas intercaladas con disoluciones de circonio muestra un pequeño aumento tras la calcinación, siendo más importante en el caso de la saponita. Se ha observado que tanto el volumen de microporos como el asociado a los mesoporos se ven afectados por el tratamiento térmico. La gran cantidad de óxido de circonio presente en las arcillas 
intercaladas, sobre todo en el caso de la saponita, ha sugerido que puede existir una relación entre las propiedades texturales desarrolladas en la calcinación a $500{ }^{\circ} \mathrm{C}$ y la cantidad de óxido de circonio presente. En este sentido hay que tener en cuenta la contribución de los microporos desarrollados por la descomposición de los ligandos acetato así como la posible presencia en la superficie externa de partículas mesoporosas de óxido de circonio.

\section{BIBLIOGRAFÍA}

1. Z. Ge, D. Li y T.J. Pinnavaia, “Preparation of Alumina-Pillared Montmorillonites with High Thermal Stability, Regular Microporosity and Lewis/Brönsted Acidity", Micropor Mater. 3, 165-175 (1994).

2. R. Toranzo, M.A. Vicente, M.A. Bañares-Muñoz, L.M. Gandía y A. Gil, "Pillaring of Saponite with Zirconium Oligomers", Micropor Mesopor Mater. 24, 173-188 (1998).

3. J.P. Sterte y J. Shabtai, “Cross-Linked Smectites. V. Synthesis and Properties of Hydroxy- Silicoaluminum Montmorillonites and Fluorhectorites", Clays Clay Miner. 35, 429-439 (1987).

4. F. Figueras, "Pillared Clays as Catalysts", Catal. Rev. Sci. Eng. 30, 457-499 (1988).

5. M.L. Occelli, "Physicochemical Properties of Pillared Clay Catalysts", Stud. Surf. Sci. Catal. 35, 101-137 (1988)

6. D. Tichit, F. Fajula, F. Figueras, B. Ducourant, G. Mascherpa, C. Gueguen y J. Bousquet, "Sintering of Montmorillonites Pillared by Hydroxy-Aluminium Species", Clays Clay Miner. 36, 369-375 (1988).

7. D.E.W. Vaughan, "Pillared Clays-A Historical Perspective", Catal. Today, 2, 187-198 (1988).

8. J.T. Kloprogge, E. Booy, J.B.H. Jansen y J.W. Geus, "The Effect of Thermal Treatment on the Properties of Hydroxy-Al and Hydroxy-Ga Pillared Montmorillonite and Beidellite", Clay Miner. 29, 153-167 (1994).

9. L.S. Cheng y R.T. Yang, "Tailoring Micropore Dimensions in Pillared Clays for Enhanced Gas Adsorption", Micropor Mater. 8, 177-186 (1997)

10. R.T. Yang y M.S.A. Baksh, "Pillared Clays as a New Class of Sorbents for Gas Separation", AIChE Journal 37, 679-686 (1991).

11. G.W. Brindley y G. Brown, en Crystal Structures of Clay Minerals and their X-Ray Identification. Mineralogical Society, London, 1984.

12. K. Suzuki, M. Horio y T. Mori, "Preparation of Alumina-Pillared Montmorillonite with Desired Pillar Population", Mater. Res. Bull. 23, 17111718 (1988).

13. M. Horio, K. Suzuki, H. Masuda y T. Mori, "Alkylation of Toluene with Methanol on Alumina-Pillared Montmorillonite. Suppression of Deactivation by Control of the Lateral Spacings of Pillars", Appl. Catal. 72, 109-118 (1991).

14. K. Suzuki, M. Horio, H. Masuda and T. Mori, "Preparation and Property of Zirconia-Pillared Montmorillonite having Different Pillar Populations", Bull. Chem. Soc. Jpn. 64, 732-734 (1991)

15. J.R. Jones y J.H. Purnell, "The Catalytic Dehydration of Pentan-1-ol by Alumina Pillared Texas Montmorillonites of Differing Pillar Density", Catal. Lett. 28, 283-289 (1994).

16. M. Sychev, V.H.J. de Beer y R.A. van Santen, "Chromia and Chromium Sulfide Pillared Clays Differing in Pillar Density", Micropor Mater. 8, 255-265 (1997).

17. S. Chevalier, R. Franck, H. Suquet, J.F. Lambert y D. Barthomeuf, “Al-Pillared Saponites. Part. 1. IR Studies", J. Chem. Soc. Faraday Trans. 90, 667-674 (1994)

18. J.F. Lambert, S. Chevalier, R. Franck, H. Suquet y D. Barthomeuf, “Al-Pillared Saponites. Part. 2. NMR Studies", J. Chem. Soc. Faraday Trans. 90, 675-682 (1994).

19. L. Bergaoui, J.F. Lambert, R. Franck, H. Suquet y J.L. Robert, “Al-Pillared Saponites. Part 3. Effect of Parent Clay Layer Charge on the IntercalationPillaring Mechanism and Structural Properties", J. Chem. Soc. Faraday Trans., 91, 2229-2239 (1995).
20. L. Bergaoui, J.F. Lambert, H. Suquet y M. Che, "Cu" on $\mathrm{Al}_{13}$-Pillared Saponites: Macroscopic Adsorption Measurements and EPR Spectra", J. Phys. Chem. 99, 2155-2161 (1995).

21. L. Bergaoui, J.F. Lambert, M.A. Vicente-Rodríguez, L.J. Michot y F. Villiéras, "Porosity of Synthetic Saponites with Variable Layer Charge Pillared by $\mathrm{Al}_{13}$ Polycations", Langmuir 11, 2849-2852 (1995).

22. L.J. Michot, F. Villiéras, J.-F. Lambert, L. Bergaoui, Y. Grillet y J.-L. Robert, "Surface Heterogeneity in Micropores of Pillared Clays: The Limits of Classical Pore-Filling Mechanisms", J. Phys. Chem. B 102, 3466-3476 (1998).

23. A. Clearfield, M.E. Kuchenmeister, K. Wade y P. Sylvester, "Pillaring of Layered Inorganic Compounds: Fundamentals “, pp. 245-262 en Synthesis of Microporous Materials, M.L. Occelli and H. Robson. Van Nostrand Reinhold, New York, 1992.

24. T.J. Pinnavaia, M.-S. Tzou, S.D. Landau y R.H. Raythatha, "On the Pillaring and Delamination of Smectite Clay Catalysts by Polioxo Cations of Aluminum", J. Mol. Catal. 27, 195-212 (1984).

25. J.P. Sterte y J.-E. Otterstedt, "Aluminum-Oxide-Pillared Montmorillonite: Effect of Hydrothermal Treatment of Pillaring Solution on the Product Structure", Stud. Surf. Sci. Catal. 31, 631-648 (1987).

26. A. Gil y M. Montes, "Analysis of the Microporosity in Pillared Clays", Langmuir 10, 291-297 (1994).

27. J.Y. Bottero, J.M. Cases, F. Fiessinger y J.E. Poirier, "Studies of Hydrolyzed Aluminum Chloride Solutions.1. Nature of Aluminum Species and Composition of Aqueous Solutions", J. Phys. Chem. 84, 2933-2939 (1980).

28. J.Y. Bottero, D. Tchoubar, J.M. Cases y F. Fiessinger, "Investigation of the Hydrolysis of Aqueous Solutions of Aluminum Chloride.2. Nature and Structure by Small-Angle X-Ray Scattering", J. Phys. Chem. 86, 3667-3673 (1982).

29. R.A. Schoonheydt, J. van den Eynde, H. Tubbax, H. Leeman, M. Stuyckens, I. Lenotte y W.E.E. Stone, "The Al Pillaring of Clays. Part I. Pillaring with Dilute and Concentrated Al Solutions", Clays Clay Miner. 41, 598-607 (1993).

30. A. Gil y M. Montes, "Evolution of the Microporous Accessibility with the Hydrolysis Degree and the Intercalation Solution Ageing Time Conditions in Aluminium-Pillared Clays", Micropor Mater. 3, 319-329 (1994).

31. S. Moreno, R. Sun Kou y G. Poncelet, "Influence of Preparation Variables on the Structural, Textural, and Catalytic Properties of Al-Pillared Saponites", J. Phys. Chem. B 101, 1569-1578 (1997)

32. K. Ohtsuka, Y. Hayashi y M. Suda, "Microporous $\mathrm{ZrO}_{2}-$ Pillared Clays Derived from Three Kinds of Zr Polynuclear Ionic Species", Chem. Mater. 5, 1823-1829 (1993)

33. H.L. Del Castillo, A. Gil y P. Grange, "Influence of the Nature of Titanium Alkoxide and of the Acid of Hydrolysis in the Preparation of TitaniumPillared Montmorillonites", J. Phys. Chem. Solids 58, 1053-1062 (1997).

34. A. Gil y M. Montes, "Effect of Thermal Treatment on Microporous Accessibility in Aluminium Pillared Clays", J. Mater. Chem. 4, 1491-1496 (1994).

35. S. Yamanaka y M. Hattori, "Iron Oxide Pillared Clay", Catal. Today, 2, 261270 (1988).

36. J.P. Sterte, J.-E. Otterstedt, H. Thulin y F.E. Massoth, “Characterization of Alumina-Montmorillonite Complexes", Appl. Catal. 38, 119-129 (1988)

37. T. Matsuda, M. Asanuma y E. Kikuchi, "Effect of High-Temperature Treatment on the Activity of Montmorillonite Pillared by Alumina in the Conversion of 1,2,4-Trimethylbenzene", Appl. Catal. 38, 289-299 (1988).

38. C. Pesquera, F. Gonzalez, I. Benito, S. Mendioroz y J.A. Pajares, "Synthesis and Characterization of Pillared Montmorillonite Catalysts", Appl. Catal. 69, 97-104 (1991).

39.- S.J. Gregg y K.S.W. Sing, en Adsorption, Surface Area and Porosity. Academic Press, London, 1991.

40. L.M. Gandía, R. Toranzo, M.A. Vicente y A. Gil, “Non-Aggressive Pillaring of Clays with Zirconium Acetate. Comparison with Alumina Pillared Clays", Appl. Catal. A: General 183, 23-33 (1999).

41. J.W. Johnson, J.F. Brody, R.M. Alexander, L.N. Yacullo y C.F. Klein, “ZirconiaPillared Tetrasilicic Fluoromica", Chem. Mater. 5, 36-42 (1993).

42. D. Plee, L. Gatineau y J.J. Fripiat, "Pillaring Processes of Smectites with and without Tetrahedral Substitution", Clays Clay Miner. 35, 81-88 (1987).

43. M.A. Vicente, M. Suárez, M.A. Bañares-Muñoz y J.M. Martín-Pozas, "Characterization of the Solids Obtained by Pillaring of Griffithite (High Iron Content Saponite) with Al-Oligomers", Clays Clay Miner. 45, 761-768 (1997). 